phenotype and the youngest had amenorrhea with no breast development (B1) and pubic hair. Furthermore, according to physical examination, deafness was not mentioned.

Conclusions According to results, it seems that clinicians should consider different presentation for pure gonadal dysgenesis with familial pattern and further evaluation is needed in malignant degeneration of the gonadal dysgenesis in the patients with 46, XX PGD.

\section{PO-0094 ANTHROPOMETRIC CHARACTERISTICS AND BONE MINERAL DENSITY IN PATIENTS WITH PHENYLKETONURIA}

${ }^{1} \mathrm{P}$ Diaz Fernandez, ${ }^{1} \mathrm{C}$ Verastegui Martinez, ${ }^{2} \mathrm{G}$ Rodríguez Martínez, ${ }^{1} \mathrm{IC}$ Garcia Jimenez. ${ }^{1}$ Pediatria, Hospital Universitario Miguel Servet, Zaragoza, Spain; ${ }^{2}$ Pediatría, Hospital Clínico Universitario Lozano Blesa, Zaragoza, Spain

\subsection{6/archdischild-2014-307384.763}

Background Phenylketonuria (PKU) treatment requires a diet restricted from natural proteins and supplemented with phenylalanine (Phe)-free L-amino acid mixtures. Growth impairment and compromised bone mass have been described.

This study aims to evaluate anthropometric characteristics and bone mineral density (BMD) in a cohort of PKU patients.

Methods We conducted a retrospective longitudinal study collecting anthropometric characteristics (weight, height, body mass index (BMI) and BMD every 6 months from birth to 12 years of age in 34patients with diet restrictions.

We compared the data results, expressed as z-scores, with the general population, as well as between patients with Phe $<360$ $\mathrm{mmol} / \mathrm{ml}$ (optimal) and patients with $>360 \mathrm{mmol} / \mathrm{ml}$.

Results Our PKU patients are shorter than the reference population; the sample mean was below $\mathrm{z}-\mathrm{score}=0$. Weight was comparable to that of the reference population and BMI had a tendency to be over the population mean.

Growth impairment in PHA-deficiency is not related to plasma Phe concentration at birth but might be related to its levels throughout the follow up; patients with $<360 \mathrm{mmol} / \mathrm{ml}$ were shorter.

BMD was below the population mean in all cases $(52 \%$ osteopenia).

Conclusions PKU children in our study have a below than average height. Weight is consistent with the population average and BMI tends to be above it.

Height seems more affected among those patients with better metabolic control.

BMD is below the population mean. These data do not vary depending on the levels of Phe at diagnosis, but by the phenylalaninemia during growth.

Further studies are needed to investigate the effect of diet restriction in PKU.

\section{PO-0095 EARLY PROGRAMMING OF AEROBIC AND NEUROMUSCULAIR FITNESS AT PRIMARY SCHOOL AGE. THE ABCD-STUDY}

${ }^{1} \mathrm{AW}$ van Deutekom, ${ }^{2} \mathrm{M}$ Chin-A-Paw, ${ }^{3} \mathrm{TGM}$ Vrijkotte, ${ }^{1} \mathrm{RJBJ}$ Gemke. ${ }^{1}$ Pediatrics and EMGO +, VU University Medical Center, Amsterdam, Netherlands; ${ }^{2}$ Public Health and EMGO+, VUmc University Medical Center, Amsterdam, Netherlands; ${ }^{3}$ Public Health, Academic Medical Center, Amsterdam, Netherlands

10.1136/archdischild-2014-307384.764
Background and aims Low birth weight and accelerated postnatal growth are associated with adult cardiovascular disease. In this perspective body composition and obesity can result from a disturbed energy balance due to early reprogramming of energy intake and expenditure. We hypothesise that low birth weight and accelerated growth may predispose ("program") reduced physical fitness at 8-9 years of age.

Methods Aerobic fitness was measured using a 20 metre multistage shuttle run test (20m-MSRT) and neuromuscular fitness using the standing broad jump (SBJ) test and handgrip strength test was measured in 194 children (104 boys) of Dutch ethnicity at mean age 8.6 years in a prospective birth cohort.

Results Subjects with low birth weight and accelerated infant growth reached mean $( \pm S D)$ 20m-MSRT levels of 3,9 which was significantly lower than (1) normal birth weight and normal infant growth (2) low birth weight and normal infant growth and (3) normal birth weight and accelerated infant growth groups (all $\mathrm{p}<0.01$ ). Low birth weight subjects had mean grip strength of $12.3 \mathrm{~kg}( \pm 3.0)$, which was significantly lower than normal weight subjects with no effect of infant growth on this relationship. There was no association of birth weight or infant growth with grip strength or SBJ.

Conclusions Low birth weight with accelerated infant weight gain was associated with diminished aerobic fitness. Higher birth weight was associated with increased neuromuscular fitness. These early changes may explain increased susceptibility to obesity and related risk factors in low birth weight and early growth accelerated children.

\section{PO-0096 CLINICAL EFFECTIVENESS OF IDURSULFASE IN BOYS AGED 0-5 YEARS WITH HUNTER SYNDROME: 3-YEAR DATA FROM THE HUNTER OUTCOME SURVEY}

${ }^{1}$ J Muenzer, ${ }^{2} \mathrm{R}$ Giugliani, ${ }^{3} \mathrm{M}$ Scarpa, ${ }^{4} \mathrm{~A}$ Tanaka, ${ }^{5} \mathrm{~A}$ Anna Tylki-Szymanska, ${ }^{6}$ Morin, ${ }^{7} \mathrm{M}$ Beck. ${ }^{1}$ Department of Paediatrics, University of North Carolina at Chapel Hill, Chapel Hill, USA; ${ }^{2}$ Medical Genetics Service, Hospital de Clínicas de Porto Alegre, Porto Alegre, Brazil; ${ }^{3}$ Rare Disease Centre, Dr. Horst Schmidt Kliniken GmbH, Wiesbaden, Germany; ${ }^{4}$ Department of Pediatrics, Osaka City University Graduate School of Medicine, Osaka, Japan; ${ }^{5}$ Department of Metabolic Diseases, The Children's Memorial Health Institute, Warsaw, Poland; ${ }^{6}$ Rare Diseases, Shire, Eysins, Switzerland; ${ }^{7}$ Department of Pediatrics, University Medical Center Johannes Gutenberg University, Mainz, Germany

\subsection{6/archdischild-2014-307384.765}

Background and aims Symptoms of Hunter syndrome typically become apparent at 2-4 years of age. Previous analyses have demonstrated improvements in certain clinical measures in young patients receiving idursulfase (Shire); however, data on long-term idursulfase use in these patients remain limited. This analysis used data available in the Hunter Outcome Survey (HOS), a global, observational registry sponsored by Shire, to investigate long-term effectiveness of idursulfase in boys with Hunter syndrome aged $0-5$ years.

Methods As of January 2014, 260/564 males followed prospectively in HOS had received $\geq 1$ idursulfase infusion (excluding those who had received a bone marrow transplant or were enrolled in the TKT018/TKT024 clinical trials), were aged 0-5 years at treatment initiation and were included in the analysis. Median age at first treatment was 3.5 years; median treatment duration was 41.6 months. Clinical measures recorded in HOS at annual timepoints over 3 years were compared with baseline values.

Results Median urinary glycosaminoglycan (uGAG) levels, liver size and left ventricular mass index had improved at all yearly 
timepoints compared with baseline (median uGAG, $-64.6 \%$ versus baseline after 3 years, $\mathrm{n}=32$ ). However, the number of patients with baseline and post-treatment data available was relatively low in some instances $(n=7-56)$, especially at 3 years.

Conclusions This analysis of data from HOS suggests that a positive trend in the clinical effectiveness of idursulfase was generally maintained over 3 years of treatment in these boys aged 0-5 years with Hunter syndrome, supporting the results of previous analyses.

\section{PO-0097 THE MOLECULAR GENETIC STUDIES FOR DETERMINATION OF LATENT MOSAICISM AND PARENTAL ORIGIN OF X CHROMOSOME IN GIRLS WITH TURNER SYNDROME IN UZBEKISTAN}

MD Mirkhaydarova, NSH Ibraghimova, RS Mukhamedov. Laboratory of Genomics, Institute of Bioorganic Chemistry Academy of Sciences Republic of Uzbekistan, Tashkent, Uzbekistan

\subsection{6/archdischild-2014-307384.766}

Goal Identification of latent mosaicism and determination of a parental origin of an $\mathrm{X}$ chromosome in TS patients in Uzbekistan.

Methods Molecular genetic studies are carried out in 35 patients with TS (26 with monosomy, 9 with mosaicism) at the age of 7 to 16 and their parents.

Results Three X-linked markers (DMD 49, AR and DX1283E) were studied on the basis of their high level of heterozygosis, a number of alleles (11 to 19) and localization both on a short and long X chromosome arm. The results obtained confirm that the use of a set of these primers (DMD 49, AR and DX1283E) will allow enhancing a probability of obtaining an informative marker and detection of latent X-mosaicism. Monozygosity on all 3 markers indicates the presence of only one X chromosome that in female patients will correspond to true monosomy. Heterozygosis of one marker suggests on the presence of an additional second $\mathrm{X}$ chromosome or a part of an X chromosome which is observed both in 46XX karyotype (healthy) and in mosaic variants of chromosomal anomalies (45X0-46XX, 45X0-46?Y).

Conclusions A comparative analysis of polymorphic markers in TS patients and their parents enable us to establish the origin of an $\mathrm{X}$ chromosome and determine in gametogenesis of which parents meiotic impairment occurred. Identification of mosaicism in Turner syndrome is very important from the viewpoint of setting correlations between a phenotype and karyotype.

\section{PO-0098 RELATION BETWEEN VISCERAL AND SUBCUTANEOUS FAT WITH BONE MINERAL DENSITY AMONG EGYPTIAN CHILDREN AND ADOLESCENTS}

SEl-Masry, NE Hassan. Biological Anthropology, National Research Centre, Giza, Egypt

\subsection{6/archdischild-2014-307384.767}

Objective To verify the influence of visceral and subcutaneous fat, on bone mineral density (BMD) among Egyptian children and adolescents.

Subjects and methods The study involved 78 children and adolescents ( 38 boys and 40 girls), aged 8-17 years old. They were divided into 2 age groups: children group aged 8-12 years and adolescent group aged from 13 up to 17 years. Anthropometric measurements, body composition, visceral and subcutaneous fat, and BMD were determined.

Results Data demonstrated significant positive relationship among children between visceral fat with BMD Z-score (total and at lumbar spines) and total BMD, while subcutaneous fat had significant positive association with bone mineral content (total and at lumbar spines), and total BMD and its Z-score. For adolescents, no association was observed between either of visceral or subcutaneous fat with any parameter of BMD.

Conclusions Visceral and subcutaneous fat had significant positive association with BMD in children; where the association was stronger with subcutaneous fat; while this association disappears in adolescents.

\section{Gastroenterology and Hepatology/Nutrition}

\section{PO-0099 LOCAL COMPLICATIONS AND ADVERSE DRUG REACTIONS IN PAEDIATRIC INFLAMMATORY BOWEL DISEASE}

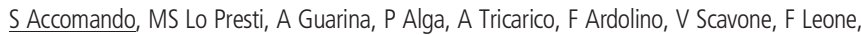
L Messina, G Corsello. Department of Sciences for Health Promotion and Mother and Infant Care "G. D'Alessandro", Pediatrics Section, Palermo, Italy

\subsection{6/archdischild-2014-307384.768}

Background and aims Complications of inflammatory bowel disease (IBD) may involve gastro-intestinal system and several other organs. Extraintestinal manifestations (EIMs) are reported in about one third of adults with IBD and they may be related or not to disease activity, or adverse effects related to therapy (ADR, Adverse Drug Reactions). In paediatric patients EIMs are yet poorly understood, with few published data. Here we describe local complications and EIMs in our paediatric series of IBD patients.

Methods Five paediatric patients (age 10-18) with complicated IBD were enrolled. Diagnosis of IBD was obtained by integrating clinical data, laboratory tests, imaging studies (Magnetic Resonance Enterography) and endoscopy. All underwent protocol therapy with azathioprine and mesalazine.

Results $80 \%$ of patients (4/5) had a Crohn's disease, 20\% (1/5) an indeterminate colitis. 4 patients $(80 \%)$ were females. Local complications reported were 2 cases of intestinal occlusion which required long hospitalisation, antibiotic therapy and total parenteral nutrition, and 1 case of bowel fistulization and formation of abscess treated with combined antibiotic and biological therapy. The most frequent EIMs were ADR: 2 cases of recurrent hypertransaminasemia due to mesalazine, 2 asymptomatic pancreatitis by azathioprine, 1 hypercortisolism with osteopenia, hypertrichosis and round face. All the reported ADR resolved spontaneously stopping the drug administration. The remaining observed EIMs were 1 case of Sweet's syndrome with spontaneous resolution and 1 case of sideropenic anaemia.

Conclusions In our patients local complications of IBD were severe and required hospitalisation with aggressive therapy; ADR were mostly due to reversible effects of treatment. This emphasises the need for more targeted therapies in children.

\section{P0-0100 CHARACTERISTICS OF SPONTANEOUS CHILDREN'S BREAST PREFERENCE: A CROSS-SECTIONAL SELF-ADMINISTERED SURVEY}

${ }^{1} \mathrm{~S}$ Al-Abdi, ${ }^{2} \mathrm{~A}$ Al-Omran, ${ }^{3} \mathrm{~S}$ Al-Omran, ${ }^{3} \mathrm{M}$ Al-Nasser, ${ }^{3} \mathrm{M}$ Al-Aamri. ${ }^{1}$ Pediatrics, King Abdulaziz Hospital, Alhassa, Saudi Arabia; ${ }^{2}$ Pediatrics, Almana General Hospital, Alhassa, Saudi Arabia; ${ }^{3}$ Pediatrics, Maternity and Children Hospital, Alhassa, Saudi Arabia

10.1136/archdischild-2014-307384.769 